\title{
Homoeopathic drug induced change in liposomal anisotropy and associated change in van't Hoff enthalpy
}

\begin{abstract}
Using liposomal membrane of 1, 2-dipalmitoyl-sn -glycero-3-phosphatidyl choline, a well accepted model for biological membrane, we have measured the change in membrane anisotropy due to incorporation of three homoeopathic drugs silicea, sulphur and calc carb and the associated values of change in Van't Hoff enthalpy have been calculated. Our experimental results reveal that these three homoeopathic drugs affect the membrane anisotropy in different ways and this change depends upon the potency of the medicine.
\end{abstract}

Volume 3 Issue $6-2018$

\author{
Debbethi Bera, Piu Das, Ruma Basu, Sukhen \\ Das, Monalisa Chakraborty, Papiya Nandy \\ Centre for Interdisciplinary Research and Education, India
}

Correspondence: Papiya Nandy, Centre for Interdisciplinary Research and Education, Kolkata 700 068, India, Email pnandy00@gmail.com

Received: November 16, 2018 | Published: December 20, 2018

\section{Introduction}

Actions of drugs are in general membrane based. However, with lot of research work performed in the field of homeopathic medicine, there is very little work done on the effect of these medicines on some physical properties of the membrane. ${ }^{1}$

With increase in temperature, the lipid mileau of the biological membrane goes through a phase transition from gel state to liquid crystalline state. External agents, like temperature, drug and chemicals can affect the membrane anisotropy, thereby affecting the efficacy of the drug. For hydrophobic agents, the molecules enter the lipid mileau and increase the anisotropy and the effect is reverse for hydrophilic agents.

In our earlier work using homeopathic medicines we have shown how some metal based homeopathic medicines monitor the membrane anisotropy. ${ }^{2}$

Using three homeopathic medicines: silicea, sulphur and calc carb, we have reported here how these medicines change anisotropy of the liposomal membrane of 1, 2-dipalmitoyl-sn -glycero-3- phosphatidyl choline (DPPC), a well accepted model for biological membrane, and calculated the associated change in Van't Hoff enthalpy. Our experimental results reveal that this effect depends upon the potency of the medicine used.

\section{Materials}

1, 2-dipalmitoyl-sn -glycero-3- phosphatidyl choline (DPPC) was purchased from Avanti Polar Lipid and was used for liposome formation. Three homeopathic drugs silicea, sulphur and calc carb each of potency $6 c, 30 c, 200 c$, prepared as per standard procedure [Homoeopathic Pharmacopoeia of India, 1971, published by Ministry of Health, Govt. of India], were obtained from HAPCO, India.

Chloroform and the fluorescent probe 1, 2 diphenyl 1, 3, 5 hexatriene (DPH) were purchased from E. Merck (India). De-ionised water was used throughout the experiment. For sonication, a bath sonicator was used. Fluorescence spectrophotometer (Spectra Max M5) was used for fluorescence measurements.

\section{Method}

\section{Preparation of liposome and drug incorporation}

$0.0001 \mathrm{gm}$ DPPC was dissolved in $5 \mathrm{ml}$ chloroform in each of the 10 round bottom flask (RBF) and was dried out to a thin film by rotary evaporator.

a. Thin films were kept overnight in desiccator. After 24 hours, $4 \mathrm{ml}$ of water and $1 \mathrm{ml}$ of 3 drugs at 3 potency were added to each RBF containing the thin film. For control $5 \mathrm{ml}$ of water was taken.

b. These samples were sonicated 5 times for 15 minutes each. The RBFs were kept at $4^{\circ} \mathrm{C}$ overnight.

c. RBF were then thawed to room temperature and sonicated for 15 minutes.

\section{Measurement of anisotropy}

$10 \mu 1$ of the fluorescent probe DPH dye was added to each of the sample.

Using emission at $428 \mathrm{~nm}$ and excitation at $360 \mathrm{~nm}$, fluorescence polarisation of embedded DPH probe molecules was used to detect the phase behaviour of the liposomal membrane of DPPC. And the variation of anisotropy with temperature has been shown for Silicea $(6,30,200 \mathrm{C}$ potency) in Figure 1.

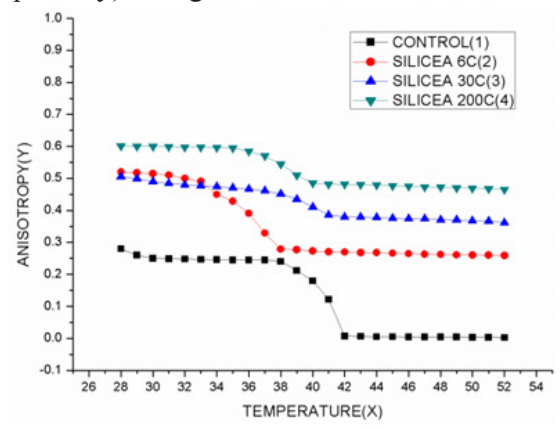

Figure I Variation of anisotropy with temperature for Silicea at different potencies, compared to the control. 


\section{Calculation of Van't Hoff enthalpy}

Following the method of Marky and Breslauer we have calculated the change in Van't Hoff enthalpy associated with phase transition between gels to liquid crystalline phase in the liposomal membrane of DPPC. ${ }^{3}$

Let us consider $\alpha$ to be equal to the fraction of lipids in the liquid crystalline state. As any fractional change in $\alpha$ denotes the extent of phase change, then anisotropy-temperature curve can be converted to the corresponding $\alpha$-temperature curve by an upper baseline and a lower baseline. By convention the phase transition temperature $\left(\mathrm{T}_{\mathrm{m}}\right)$ corresponds to the temperature for which the value of $\alpha$ is $0.5{ }^{4}$

The van't Hoff enthalpy can be calculated from the relation

$$
\Delta H=R T^{2}[d(\ln K) / d T]_{T=T m}(1)
$$

Where $\mathrm{R}$ is the gas constant and $\mathrm{K}$ is the equilibrium constant. The phase transition from gel to liquid crystalline phase is an endothermic process and hence $\Delta \mathrm{H}$ is positive.

For complete evaluation of $\Delta \mathrm{H}, \mathrm{K}$ must be expressed in terms of $\alpha$. Assuming that each lipid molecule can exist either in the gel state or in the liquid crystalline state, the transition will proceed in a twostate manner. The value of the equilibrium constant depends on the molecularity of the transition, $\mathrm{n}$, which in this case is equal to unity.

In terms of $\alpha, \Delta H$ at the phase transition temperature, $T_{m}$, can be written as

$$
\left.\Delta H\right|_{T m}=R\left(T_{m}\right)^{2}(2+2 n)[\delta \alpha / \delta T]_{T=T m}
$$

For $\mathrm{n}=1$, this equation reduces to

$$
\left.\Delta H\right|_{T m}=4 R\left(T_{m}\right)^{2}[\delta \alpha / \delta T]_{T=T m}
$$

We have calculated the van't Hoff transition enthalpy in all the four liposomal system. Figure 2 shows the comparative values of $\Delta \mathrm{H}$ for Silicea.

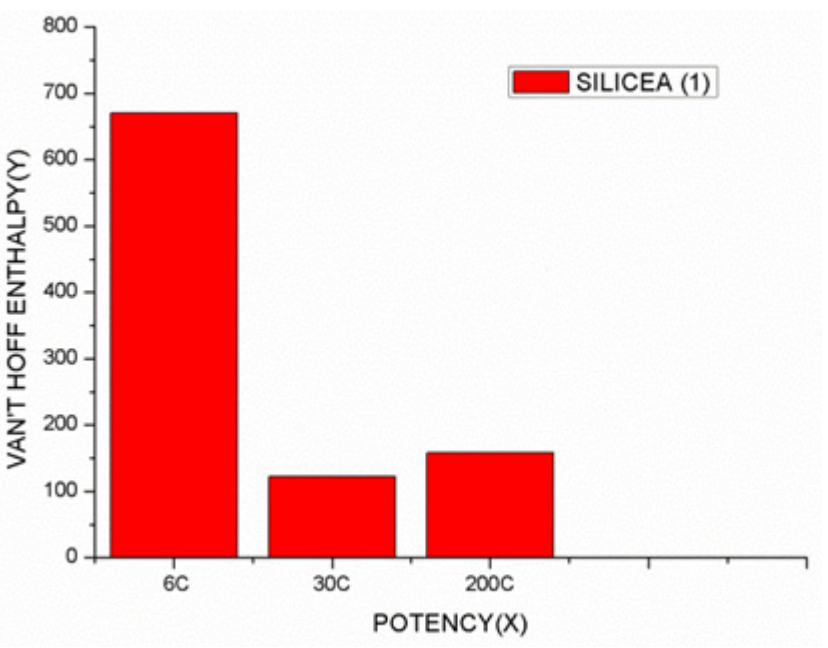

Figure 2 Variation of enthalpy with potency for Silicea.

\section{Results}

Table 1.
Table I shows the comparative values of van't Hoff Enthalpy for different medicines at three different potencies

\begin{tabular}{ll}
\hline Sample & van't Hoff Enthalpy KJ/mol \\
\hline Control & 1121.66 \\
Silicea 6C & 670.16 \\
Silicea 30C & 122.7 \\
Silicea 200C & 158.1 \\
Sulphur 6C & 156.4 \\
Sulphur 30C & 57.84 \\
Sulphur 200C & 42.74 \\
Calc carb 6C & 33.02 \\
Calc carb 30C & 28.09 \\
Calc carb 200C & 193.64 \\
\hline
\end{tabular}

\section{Conclusion}

Our result shows that Van't Hoff enthalpy $(\Delta \mathrm{H})$ decrease in all cases compared to the control i.e. for the sample without any drug. The decrease is maximum in case of Calc carb.

The two drugs Silicea and Sulphur get attached to the interior core of the hydrophobic tail region of the liposome and thus their contribution to the increase in membrane anisotropy is significant and more heat is required for phase transition of the lipid mileau. On the other hand, as the drug Calc carb gets attached to a region nearer to the hydrophilic head group its contribution to the decrease in membrane anisotropy is more and hence less heat is required for phase transition of the lipid mileau. This fact is manifested in the Van't Hoff enthalpy change.

As the potency increases, i.e.as the size of the drug associate decreases, the value of $\Delta \mathrm{H}$ decreases for the drugs Silicea and Sulphur. This picture is reversed in case of Calc carb.

\section{Acknowledgments}

None.

\section{Conflicts of interest}

Author declares there is no conflict of interest.

\section{References}

1. Nandy P. A review article of basic research on homeopathy from a physicist's point of view. Ind J Research in Homeopathy. 2015;9(3):141151.

2. Ghosh S, Charaborty M, Das S, et al. Effect of different potencies of nano medicine Cupurum metallium on membrane fluidity- a biophysical study. Am J Homeopathic Medicine. 2014;107(4):161-169.

3. De S, Ghosh AK, Basu R, et al. Calculation of van't Hoff enthalpy associated with aspirin-induced change in liposomal membrane anisotropy. Physics in Medicine and Biology. 1996;41:383-386.

4. Marky LA, Breslauer KJ. Calculating thermodynamic data for transitions of any molecularity from equilibrium melting curves. Biopolymer. 1387;26(9):1601-1620. 